\title{
Avant-propos de la deuxième édition, 1999
}

Je suis heureux rétrospectivement d'avoir décidé d'écrire Vertigineuses symétries. Au cours d'une visite à l'université du Texas en 1984, je bavardais avec l'éminent physicien Steven Weinberg lorsque sa secrétaire lui apporta un courrier, qui se trouvait contenir une recension de son deuxième livre de vulgarisation. De façon naturelle, notre conversation aborda l'écriture des livres de vulgarisation en physique. Plusieurs physiciens m'avaient enouragé à écrire un manuel, sur la théorie quantique des champs en particulier. Au lieu de poursuivre dans cette direction, non seulement Weinberg m'encouragea à écrire un livre de vulgarisation, mais il me présenta à son éditeur et me fournit des conseils avisés. Ainsi, quelques mois plus tard, je me retrouvai à New-York pour déjeuner avec l'éditeur de Weinberg. Il m'avait demandé d'apporter un chapitre comme échantillon, et je choisis le chapitre sur les lois de conservation ; il commençait par la phrase : «Il n'y a pas de déjeuner gratuit. » L'éditeur se mit à rire : « $\mathrm{Si}$, il y en a. » Depuis lors, j'ai été invité à déjeuner à maintes reprises par divers éditeurs et agents, et j'ai réappris qu'en un certain sens il n'y a pas de déjeuner gratuit. Le lecteur curieux trouvera cette phrase sur le déjeuner gratuit dans le chapitre 8 , mais elle ne figure plus au début du chapitre.

Je suis heureux d'avoir écrit Vertigineuses symétries en raison de toutes les lettres extraordinaires, et même les cadeaux, que j'ai reçus de lecteurs reconnaissants, en raison de toutes ces recensions flatteuses pour l'ego, en raison du plaisir d'entendre mes mots lus par un acteur professionnel dans une cassette audio pour aveugles, et parce que j'ai vu ma prose immortelle traduite dans plusieurs langues étrangères. Mais avant tout, je suis heureux parce que ce livre m'a permis de quitter pour une fois la communauté des physiciens. J'ai été invité à donner des conférences dans toutes sortes d'endroits intéressants, par exemple au Centre National d'Arts de Bombay, où j'ai appris le rôle des symétries dans la danse indienne, et à l'Académie des Arts de Berlin où je fus invité à participer 
à un symposium sur le racisme (ce que la symétrie a à voir avec le racisme n'est pas clair pour moi). Vertigineuses symétries a lancé ma carrière d'écrivain. Les pages que j'ai extraites de mon manuscrit sont devenues le cœur de mon second livre de vulgarisation An Old's Man Toy. Il n'a pas fallu longtemps pour que je trouve des pages de Vertigineuses symétries citées dans un manuel universitaire sur l'écriture, et j'ai été invité à parler devant des auditoires sur l'écriture créative.

Je déplore que Macmillan Publishing Company ait laissé mon livre s'épuiser. Par la suite, je me suis familiarisé avec quelques statistiques désastreuses sur l'industrie commerciale de publication. Aussi suis-je heureux, particulièrement comme ancien étudiant de Princeton, que Princeton University Press publie une nouvelle édition de Vertigineuses symétries. Je remercie les physiciens Murph Goldberger, Dave Spergel et Sam Treiman qui ont encouragé ce projet, et mes éditeurs Trevor Lipscombe et Donna Krone Meyer pour leur excellent travail.

Je voudrais remercier Joe Polchinski et Roger Shepard pour leur lecture de la postface ajoutée à cette édition de Princeton University Press. Je pense qu'il est approprié de remercier à nouveau les physiciens qui ont relu tout ou partie du manuscrit : Bill Bialek, Sidney Coleman, Murray Gell-Mann, Tsung-Dao Lee, Heinz Pagels, Steve Weinberg et Franck Wilczek. Enfin je dois remercier Gretchen Zee pour des années de soutien et d'affection.

Santa Barbara, mars 1999 


\section{Remerciements}

Tout d'abord et avant tout, je souhaite remercier mon épouse Gretchen. Ses commentaires critiques incisifs, aussi bien que son soutien affectueux, ont été essentiels. Elle lisait chaque chapitre au fur et à mesure de leur écriture, tailladant brutalement dans le manuscrit. " Je ne peux pas comprendre cela! », et elle gribouillait sur la page. Et je devais revenir à la table de travail!

Nos amis Kim Beeler, Chris Groesbeck, Martha et Franck Retman et Diane Shuford ont lu différentes parties du manuscrit afin de s'assurer que le texte soit compréhensible pour le lecteur novice.

Heins Pagels et Seve Weinberg, deux éminents collègues qui ont publié des ouvrages de vulgarisation en physique, m'ont tous les deux encouragé à poursuivre mon idée d'en écrire un sur les symétries. Ils m'ont généreusement donné des conseils sur différents aspects de l'écriture et de la publication, et m'ont présenté à leurs amis du monde de l'édition.

Je suis reconnaissant à Tsung-Dao Lee, Heinz Pagels et Steve Weinberg pour leur lecture du manuscrit ainsi que pour leurs commentaires utiles et encourageants. Je voudrais aussi remercier Sidney Coleman et Franck Wilczek pour leur lecture du chapitre 12, Murray Gell-Mann pour sa lecture du chapitre 11 et Bill Bialek pour sa relecture des épreuves.

J'ai eu la chance d'avoir Charles Levine comme directeur de collection. Ses conseils et son soutien ont été indispensables. Il m'a rassuré quand j'avais besoin de l'être et critiqué quand c'était nécessaire. Je suis heureux de m'en être fait un ami.

Ma relectrice, Catherine Shaw, a accompli à l'évidence un très bon travail car j'ai dû passer presque deux mois à récrire le manuscrit pour répondre à tous ses commentaires. «Je ne peux pas comprendre cela! » disait-elle à son tour. En conséquence, le livre est devenu plus clair. Le manuscrit a été encore poli par ma responsable éditoriale Roberta Frost.

Martin Hessler m'a fourni des conseils utiles au tout début de ce projet.

J'ai aussi bénéficié des conseils de mes agents, John Brockman et Katinka Matson. 
Les artistes cités ci-dessous m'ont aidé à rendre le livre plus attirant visuellement et plus clair : Bonnie Bright, Michael Cullen, Ji-jun Huang, Eric Junker, Joe Karl, Peggy Royster, Clara Weis, Gretchen Zee.

Je suis heureux que la directrice de conception du livre ait été Helen Mills, dont le frère apparaîtra au chapitre 12. Un goût pour les symétries et l'équilibre semble être un trait de famille.

Enfin, je voudrais remercier Debra Witmoyer, Lisa Lopez, Gwenn Cattron, Katie Doremeus, Karen Murphy and Kresha Warnok qui ont dactylographié différentes parties du manuscrit. 


\section{Note du traducteur}

Le lecteur français sera sans doute surpris, et peut-être même un peu agacé, par les multiples références à Dieu, au Créateur, à l'Architecte Ultime, etc. qu'il trouvera dans le texte au fil de sa lecture. L'auteur s'en explique dans un paragraphe de la postface (page 307), « Déisme face au théisme», et je renvoie le lecteur à ce paragraphe pour plus d'explications. J'aurais pu utiliser un terme unique, par exemple "Concepteur Ultime », mais j'ai préféré restituer dans la traduction la variété des termes utilisés par l'auteur.

La première édition anglaise du livre date de 1986, et sa version révisée de 1999. En près de vingt ans la physique fondamentale a sensiblement évolué et j'ai effectué une mise à jour partielle, en particulier dans le domaine de la physique des particules élémentaires. Ainsi j'ai mentionné la découverte du boson de Higgs en 2012 au CERN, la confirmation au début des années 2000 du phénomène des oscillations neutrino et ses implications pour les masses de ces particules, la mise en évidence directe en 2012 de la violation de l'invariance par renversement du temps, etc. Cette actualisation a été effectuée principalement dans les notes en fin de livre. Il aurait été souhaitable de mettre à jour le chapitre 15 sur la grande unification et le chapitre 18 sur les cordes et supercordes, mais une telle actualisation allait au-delà de mes compétences. Toutefois mon regret de ne pas avoir pu le faire est tempéré par le fait qu'il n'existe pas de véritable consensus sur les sujets traités dans ces deux chapitres, et que la plupart des idées nouvelles sont encore très spéculatives. Je ne peux que renvoyer le lecteur à des livres récents, par exemple ceux de Sean Carroll : The Big Picture, de Lisa Randall : Knocking on Heaven's Door, ou de Helen Quinn et Yossi Nair : The Mystery of the Missing Antimatter, qui sont cités dans les notes $13 \mathrm{f}$ et 14g.

Michel Le Bellac, professeur émérite de physique 
